\title{
On Projective Equivalence of Univariate Polynomial Subspaces
}

Peter CROOKS ${ }^{\dagger}$ and Robert MILSON $\ddagger$

${ }^{\dagger}$ Department of Mathematics, University of Toronto, Toronto, Ontario, Canada M5S 2E4

E-mail: peter.crooks@utoronto.ca

$\ddagger$ Department of Mathematics and Statistics, Dalhousie University, Halifax, Nova Scotia, Canada B3H 3J5

E-mail: rmilson@dal.ca

Received June 05, 2009, in final form December 03, 2009; Published online December 06, 2009 doi:10.3842/SIGMA.2009.107

\begin{abstract}
We pose and solve the equivalence problem for subspaces of $\mathcal{P}_{n}$, the $(n+1)$ dimensional vector space of univariate polynomials of degree $\leq n$. The group of interest is $\mathrm{SL}_{2}$ acting by projective transformations on the Grassmannian variety $\mathcal{G}_{k} \mathcal{P}_{n}$ of $k$-dimensional subspaces. We establish the equivariance of the Wronski map and use this map to reduce the subspace equivalence problem to the equivalence problem for binary forms.
\end{abstract}

Key words: polynomial subspaces; projective equivalence

2000 Mathematics Subject Classification: 14M15; 15A72; 34A30; 58K05

\section{Introduction}

Given a representation of a Lie group $G$ on a finite-dimensional vector space $V$, it is natural to consider the associated action of $G$ on $\mathcal{G}_{k} V$, the Grassmannian variety of $k$-dimensional subspaces of $V$. This non-linear $G$ action is realized via the so-called Plücker embedding of $\mathcal{G}_{k} V$ into the projectivization of $\Lambda^{k} V$, the $k^{\text {th }}$ exterior product of $V$. The following question is of central importance:

When are two subspaces $U_{1}, U_{2} \in \mathcal{G}_{k} V$ equivalent up to a $G$-action?

The present article is devoted to a specific instance of this general equivalence problem. Here, the group of interest is $\mathrm{SL}_{2}$ acting on

$$
\mathcal{P}_{n}(z):=\operatorname{span}\left\{1, z, z^{2}, \ldots, z^{n}\right\},
$$

by fractional linear transformations. Classical invariant theory $[13,17]$ is concerned with the equivalence problem for one-dimensional subspaces; i.e., binary forms, defined up to scalar multiple. We are interested in the following generalization.

Definition 1.1. Let $U, \hat{U} \in \mathcal{G}_{k} \mathcal{P}_{n}$ be polynomial subspaces with respective bases $p_{i}, \hat{p}_{i} \in \mathcal{P}_{n}$, $i=1, \ldots, k$. We say that the two subspaces are projectively equivalent, and write $U \sim \hat{U}$ if there exist a fractional linear transformation

$$
z=\frac{a \hat{z}+b}{c \hat{z}+d}, \quad a d-b c=1,
$$

and an invertible $k \times k$ matrix $A_{i j}$ such that

$$
\hat{p}_{i}(\hat{z})=(c \hat{z}+d)^{n} \sum_{j=1}^{k} A_{i j} p_{j}\left(\frac{a \hat{z}+b}{c \hat{z}+d}\right), \quad i=1, \ldots, k .
$$


As we shall see, the equivalence problem for polynomial subspaces furnishes interesting analogues and extensions of certain concepts from the classical invariant theory of binary forms.

Our original motivation for studying the equivalence problem for polynomial subspaces relates to the theory of differential operators with invariant polynomial subspaces [7]. The two fundamental questions in this area are: (1) to characterize polynomial subspaces that are left invariant by a 2 nd order differential operator; and (2) to characterize infinite polynomial flags preserved by a 2nd order operator. The first question is central to the theory of quasi-exact solvability in quantum mechanics [9]. The second question has interesting applications to orthogonal polynomials [11]. Invariance with respect to a differential operator is an $\mathrm{SL}_{2}$-invariant property, and further progress depends crucially on advancing the understanding of invariant properties of polynomial subspaces. The present paper gathers the necessary exposition and theorems. We plan to return to the question of differential operators in subsequent publications.

The main tool in our analysis of the subspace equivalence problem is the Wronskian operator. Let $p_{1}, \ldots, p_{k} \in \mathcal{P}_{n}$ be linearly independent polynomials and $W=\mathrm{W}\left(p_{1}, \ldots, p_{k}\right)$ the corresponding Wronskian determinant. It isn't hard to show that $\operatorname{deg} W \leq k \ell$, where $\ell=n+1-k$ is the codimension of a $k$-dimensional subspace of $\mathcal{P}_{n}$. Since equality holds generically, we can define the linear Wronski map W: $\Lambda^{k} \mathcal{P}_{n} \rightarrow \mathcal{P}_{k \ell}$, and by restriction its non-linear analogue ${ }^{1}$ $\mathrm{W}: \mathcal{G}_{k} \mathcal{P}_{n} \rightarrow \mathcal{G}_{1} \mathcal{P}_{k \ell}$. For details, see Proposition 4.2 and the extensive discussion in [19]. The Wronski map is intimately related to the Schubert calculus [8] and the enumeration of rational curves in projective space [14], and has received a great deal of recent attention owing to its connection with the exactly solvable Gaudin model and the Shapiro conjecture; see $[4,19,16]$ and the references therein.

Key to our analysis is the fact that the the Wronski map is $\mathrm{SL}_{2}$ equivariant. From the representation theory point of view, $\mathcal{P}_{k \ell}$ is just the highest weight component of the decomposition of $\Lambda^{k} \mathcal{P}_{n}$ into irreducible $\mathrm{SL}_{2}$ representations. Reference [1] explores this point of view, as well as the relationship of the Wronskian to classical invariant theory. In classical terminology, the Wronskian is just a particular combinant, that is a joint covariant of polynomials $p_{1}, \ldots, p_{k}$ that enjoys a determinantal transformation law with respect to a change of basis. The projections of $\Lambda^{k} \mathcal{P}_{n}$ onto the irreducible $\mathrm{SL}_{2}$ components is accomplished by a sequence of Wronskianlike combinants $C_{i}: \Lambda^{k} \mathcal{P}_{n} \rightarrow \mathcal{P}_{k \ell-2 i}$ where $i=0,2, \ldots, k$ and where $C_{0}$ is just the ordinary Wronskian. It is shown in [1] that the mapping

$$
\operatorname{span}\left(p_{1}, \ldots, p_{k}\right) \mapsto\left[\left(C_{0}, C_{2}, \ldots, C_{k}\right)\right]
$$

defines an equivariant projective embedding of the Grassmannian $\mathcal{G}_{k} \mathcal{P}_{n}$ into a projective space of sufficiently high dimension. Therefore, in principle, the equivalence problem for polynomial subspaces is fully solved by considering the joint $\mathrm{SL}_{2}$ equivalence problem [18] for the combinants $\left(C_{0}, C_{2}, \ldots, C_{k}\right)$.

Our main observation is that the equivalence problem for polynomial subspaces can be solved by considering just $C_{0}$, the ordinary Wronskian. The equivariance of the Wronski map means that if two subspaces are projectively equivalent, then so are their respective Wronskians. The converse, of course, is not true. The consequence of discarding the other combinants is that the resulting mapping is no longer one-to-one. Indeed, the degree of the Wronski map $U \mapsto$ $W(U)=C_{0}$ is of central concept in the Schubert calculus and has an important combinatorial interpretation $[8,12]$. However, we can assert the following.

\footnotetext{
${ }^{1}$ We use the same symbol $\mathrm{W}$ for both maps and for the Wronskian operation. Ambiguity isn't a danger, because the particular choice of usage is indicated by the context.
} 
Proposition 1.2. Let $U, \hat{U} \in \mathcal{G}_{k} \mathcal{P}_{n}$ be polynomial subspaces such that $W(\hat{U})=g \cdot W(U)$ where $g \in \mathrm{SL}_{2} \mathbb{C}$ is a projective transformation. Let us furthermore suppose that $\mathrm{Stab} W(U)$, the subgroup of projective transformation that leaves $W(U)$ invariant, is trivial. Then $U \sim \hat{U}$ if and only if $\hat{U}=g \cdot U$.

Thus, in the generic case, we can decide the equivalence problem for polynomial subspaces $U, \hat{U}$ as follows. First we decide whether the corresponding Wronskians are equivalent. If the Wronskians are inequivalent, then so are $U$ and $\hat{U}$. However, if the Wronskians are equivalent, and if we can determine the fractional linear transformation that relates $W(U)$ and $W(\hat{U})$, then we can also decide the question of equivalence of the subspaces $U$ and $\hat{U}$.

The classical approach to the equivalence of binary forms is based on an integrity basis a finite generating set of invariants or covariants [15]. Here, we follow a different, more modern approach based on moving frames introduced in [17]. It turns out that if a polynomial has a finite symmetry group, then it can be fully characterized by two absolute rational covariants. In [3] the equivalence problem for binary forms is reduced to a procedure that tests the compatibility of these two covariants. In the case of a positive outcome of the equivalence test, the procedure actually determines the projective transformation $g \in \mathrm{SL}_{2} \mathbb{C}$ that relates the binary forms in question. If we apply the algorithm of [3] to Wronskians $W(U)$ and $W(\hat{U})$ and find a transformation $g$ such that $W(\hat{U})=g \cdot W(U)$, then, in the generic case, we can test for the equivalence of $U$ and $\hat{U}$ as per the above Proposition. The case where the Wronskian has an infinite symmetry group can also be handled by means of covariants. See Section 5 for the details.

Here is the outline of the rest of the paper. Apolarity is another key notion in the classical invariant theory [15]. The corresponding, and closely related, notion for polynomial subspaces is equivariant duality between dimension and codimension. The duality is defined and discussed in Section 2. Invariance of the Wronski map with respect to the apolar duality is established in Theorem 4.7. This theorem is far from surprising, and the proof is straightforward; however, we were unable to locate the theorem in the literature.

Section 3 gathers the necessary background on partitions, reduced row echelon form, and canonical reduced bases. Section 4 is devoted to the proof of the equivariance of the Wronski map. These sections are largely expository, but we include them for the convenience of the reader. An explicit proof of equivariance of the Wronskian can be found in [1]. In the present paper, we present two alternative, elementary proofs of equivariance. The first proof is novel (again, to our best knowledge,) while the second builds on a proof sketch in [4].

Section 5 is devoted to the equivalence problem for polynomial subspaces. There we present a number of novel (to our best knowledge) results. Theorem 5.4 concerns the reduction of the the equivalence problem for subspaces to the equivalence problem for binary forms in the case of a finite symmetries. Theorem 5.7 deals with the non-generic case of infinite symmetry groups. We prove that a subspace $U$ has an infinite symmetry group if and only if, up to a fractional linear transformation, it is spanned by monomials. Equivalently, we can characterize such subspaces by a condition involving the Hessian of the Wronskian and another covariant.

Theorem 5.17 concerns the question of top powers. The classical Waring problem for polynomials asks for the smallest integer $s \geq 1$ such that a $p \in \mathcal{P}_{n}$ can be written as $s$-term sum of $n$th powers. An analogous problem for polynomial subspaces is formulated in [2]. The authors of that paper also study the following, related question: for $s \geq k$ describe the closure of the locus in $\mathcal{G}_{k}\left(\mathcal{P}_{n}\right)$ formed by subspaces that are spanned by at most $s$ top powers. We limit ourselves to the case $s=k$ and focus on the following question: to describe a decision procedure that determines whether a $k$-dimensional polynomial subspace $U \in \mathcal{G}_{k} \mathcal{P}_{n}$ admits a basis of $n$ th, or top powers. An algorithmic criterion for this condition is presented in Theorem 5.17. Finally, in Proposition 5.19 we present a striking necessary condition for the existence of a basis of top powers and conjecture that this condition is also sufficient. A proof of the conjecture is given for the cases of codimension 1 and codimension 2 subspaces. 


\section{Apolar duality}

For convenience, we work over $\mathbb{C}$, the field of complex numbers. The group $\mathrm{SL}_{2} \mathbb{C}$ is the group of unimodular transformations of $\mathbb{C}^{2}$ :

$$
\left(\begin{array}{l}
\hat{x} \\
\hat{y}
\end{array}\right)=\left(\begin{array}{ll}
a & b \\
c & d
\end{array}\right)\left(\begin{array}{l}
x \\
y
\end{array}\right), \quad a d-b c=1 .
$$

Setting $z=x / y$ we obtain the fractional linear transformation

$$
z=\frac{a \hat{z}+b}{c \hat{z}+d}, \quad a d-b c=1
$$

of complex projective space $\mathbb{C} \mathrm{P}^{1}$. We identify the latter with the extended complex plane, $\hat{\mathbb{C}}:=\mathbb{C} \cup\{\infty\} \cong \mathbb{C} \mathrm{P}^{1}$. The set of all such transformations forms the quotient group $\mathrm{PSL}_{2} \mathbb{C} \simeq$ $\mathrm{SL}_{2} \mathbb{C} /\{ \pm I\}$. Fractional linear transformations also realize the $(n+1)$ dimensional irreducible representation of $\mathrm{SL}_{2} \mathbb{C}$ by means of the following action on $\mathcal{P}_{n}$ :

$$
p(z) \mapsto \hat{p}(\hat{z})=(c \hat{z}+d)^{n} p(z), \quad p \in \mathcal{P}_{n} .
$$

Let $\mathcal{G}_{k} \mathcal{P}_{n}$ denote the Grassmannian variety whose elements are $k$-dimensional subspaces of the $n+1$ dimensional vector space $\mathcal{P}_{n}$. In our analysis of this problem, we introduce the nondegenerate bilinear form $\gamma: \mathcal{P}_{n} \times \mathcal{P}_{n} \rightarrow \mathbb{C}$, defined by the relations:

$$
n ! \gamma\left(\frac{z^{j}}{j !}, \frac{z^{k}}{k !}\right)= \begin{cases}(-1)^{j}, & \text { if } j+k=n, \\ 0, & \text { otherwise. }\end{cases}
$$

Equivalently, we can write

$$
\gamma^{-1}=\sum_{j=0}^{n}(-1)^{j}\left(\begin{array}{l}
n \\
j
\end{array}\right) z^{j} \otimes z^{n-j} .
$$

Note that $\gamma$ is symmetric if $n$ is even, and skew-symmetric if $n$ is odd. Also note that, up to sign, $\gamma(p, q)$ is the classical apolar invariant of two polynomials $p, q \in \mathcal{P}_{n}$, also known as the lineo-linear invariant [15, Section 5]. This remark establishes the following result. A direct proof is also given in [10].

Proposition 2.1. The above-defined bilinear form $\gamma$ is $\mathrm{SL}_{2} \mathbb{C}$-invariant.

Definition 2.2. For a polynomial subspace $U \in \mathcal{G}_{k} \mathcal{P}_{n}$ we define

$$
U^{*}=\left\{u \in \mathcal{P}_{n}: \gamma(u, v)=0 \text { for all } v \in U\right\},
$$

and refer to $U^{*}$ as the apolar dual.

Proposition 2.3. The apolar duality mapping $\mathcal{G}_{k} \mathcal{P}_{n} \rightarrow \mathcal{G}_{n+1-k} \mathcal{P}_{n}$ given by

$$
U \mapsto U^{*}, \quad U \in \mathcal{G}_{k} \mathcal{P}_{n}
$$

is $\mathrm{SL}_{2}$-equivariant.

Also observe that if $p_{1}, \ldots, p_{k}$ is a basis of $U$, then a basis for $U^{*}$ is obtained by solving a system of $k$ homogeneous linear equations in $n+1$ variables, namely

$$
\gamma\left(p_{j}, q\right)=0, \quad j=1, \ldots, k
$$

where $q \in \mathcal{P}_{n}$ is the unknown. 
Classically, two polynomials of degree $n$ that satisfy $\gamma(p, q)=0$ are called apolar ${ }^{2}$. The vector space of binary forms apolar to a given $p \in \mathcal{P}_{n}$ is a fundamental object in classical invariant theory and is closely related to the question of sums of $n$th powers; see Kung and Rota [15] for details. Indeed we have the following classical result.

Proposition 2.4. Suppose that $p \in \mathcal{P}_{n}$ has $n$ distinct, finite roots. Then, the $n$ dimensional dual vector space $\operatorname{span}\{p\}^{*}$ is spanned by nth powers of the form $\left(z-r_{i}\right)^{n}$ where $r_{1}, \ldots, r_{n}$ are the roots of $p(z)$.

We will return to the question of $n$th powers in the final section of our paper.

\section{Partitions, diagrams, and reduced echelon form}

\subsection{Partitions}

A partition of an integer $N \geq 0$ is a finite multi-set ${ }^{3}$ of positive integers that sum to $N$. For our purposes, it will be convenient to define a partition as a non-increasing sequence $\boldsymbol{\lambda}=\left\{\lambda_{i}\right\}$ of non-negative integers $\lambda_{1} \geq \lambda_{2} \geq \cdots \geq 0$ such that

$$
N(\boldsymbol{\lambda})=\sum_{i} \lambda_{i}<\infty
$$

We will refer to $N$ as the partition total, and call

$$
h(\boldsymbol{\lambda})=\max \left\{i: \lambda_{i}>0\right\}, \quad w(\boldsymbol{\lambda})=\lambda_{1},
$$

respectively, the height and the width. The former names the cardinality of the multi-set, while the latter names the largest element of the multi-set. The height and width terminology derives from the fact that a partition can be visualized by means of a Ferrer's diagram: $\lambda_{1}$ dots in the first row, $\lambda_{2}$ dots in the 2 nd row, etc. The trivial partition is one where $\lambda_{i}=0$ for all $i$; this corresponds to the empty multi-set. By agreement, the trivial partition has $h=w=N=0$.

Definition 3.1. Let $\boldsymbol{\lambda}=\left\{\lambda_{i}\right\}$ be a partition. For $j \geq 1$, define $\lambda_{j}^{*}$ to be the cardinality of the set $\left\{i \geq 1: \lambda_{i} \geq j\right\}$. Call the resulting sequence $\boldsymbol{\lambda}^{*}=\left\{\lambda_{j}^{*}\right\}$ the conjugate partition of $\boldsymbol{\lambda}$.

Proposition 3.2. Let $\boldsymbol{\lambda}$ be a partition of height $h$ and width $w$. The conjugate partition $\boldsymbol{\lambda}^{*}=$ $\left\{\lambda_{j}^{*}\right\}$ is the unique partition of height $w$ and width $h$ such that for all $i, j \geq 1$ we have

$$
j \leq \lambda_{i} \quad \text { if and only if } i \leq \lambda_{j}^{*} .
$$

Furthermore, conjugate partitions have the same total: $N(\boldsymbol{\lambda})=N\left(\boldsymbol{\lambda}^{*}\right)$.

In other words, the Ferrer's diagram of the conjugate partition $\lambda^{*}$ is obtained by transposing the left-aligned Ferrer's diagram of $\boldsymbol{\lambda}$; columns become rows, and vice versa. In the next section, we will characterize the apolar duality relation between polynomial subspaces using the conjugate partition. First, we characterize conjugate partitions in terms of complementary subsets.

From now on, we fix integers $k, \ell \geq 0$ and set

$$
n=k+\ell-1 \text {. }
$$

We will say that a partition is $k \times \ell$ bounded if $h \leq k$ and $w \leq \ell$. In other words, for $k, \ell \geq 1$, the Ferrer's diagram of a $k \times \ell$ bounded partition is a subset of the discrete $k \times \ell$ rectangle. Let $\mathrm{B}_{k, \ell}$

\footnotetext{
${ }^{2}$ The classical definition of apolarity for polynomials of different degrees $m>n$ involves a bilinear bracket $\mathcal{P}_{m} \times \mathcal{P}_{n} \mapsto \mathcal{P}_{m-n}$. The classical bracket matches our form $\gamma$ only in the case where $m=n$.

${ }^{3}$ The empty set corresponds to $N=0$.
} 
denote the set of all $k \times \ell$ bounded partitions. Thus, the conjugation operation $\boldsymbol{\lambda} \rightarrow \boldsymbol{\lambda}^{*}$ defines a bijection of $\mathrm{B}_{k, \ell}$ and $\mathrm{B}_{\ell, k}$. We say that a partition $\boldsymbol{\lambda}=\left\{\lambda_{i}\right\}$ is rectangular if every non-zero $\lambda_{i}$ is equal to $\lambda_{1}$. The $k \times \ell$ rectangular partition is the "largest" element of $\mathrm{B}_{k, \ell}$.

Let $\mathrm{P}_{k, \ell}$ denote the set of all strictly increasing $k$-element sequences ${ }^{4}$ in $\{0,1, \ldots, n\}$. Thus, $\boldsymbol{\nu} \in \mathrm{P}_{k, \ell}$ refers to a $k$-element sequence $0 \leq \nu_{1}<\cdots<\nu_{k} \leq n$. We now describe two useful bijections of $\mathrm{B}_{k, \ell}$ and $\mathrm{P}_{k, \ell}$. For a bounded partition $\boldsymbol{\lambda} \in \mathrm{B}_{k, \ell}$ define $\boldsymbol{\lambda}^{+}, \boldsymbol{\lambda}^{-} \in \mathrm{P}_{k, \ell}$ by setting

$$
\begin{aligned}
& \lambda_{i}^{+}=\ell-1-\lambda_{i}+i, \quad i=1, \ldots, k, \\
& \lambda_{i}^{-}=\lambda_{k+1-i}+i-1=n-\lambda_{k+1-i}^{+}, \quad i=1, \ldots, k .
\end{aligned}
$$

Observe that $\lambda_{1}^{+}=\ell-\lambda_{1}, \lambda_{k}^{+}=n-\lambda_{k}$. Similarly, $\lambda_{1}^{-}=\lambda_{k}, \lambda_{k}^{-}=\lambda_{1}+k-1$. Therefore the mappings $\boldsymbol{\lambda} \mapsto \boldsymbol{\lambda}^{+}$and $\boldsymbol{\lambda} \mapsto \boldsymbol{\lambda}^{-}$are bijective.

In addition to conjugation, bounded partitions enjoy another kind of duality, one that relates $\boldsymbol{\lambda}^{+}$and $\boldsymbol{\lambda}^{-}$. Given a bounded partition $\boldsymbol{\lambda} \in \mathrm{B}_{k, \ell}$, set

$$
\lambda_{i}^{\prime}:=\ell-\lambda_{k+1-i}, \quad i=1, \ldots, k, \quad \lambda_{i}^{\prime}:=0, \quad i>k
$$

and call the resulting partition $\boldsymbol{\lambda}^{\prime}=\left\{\lambda_{i}^{\prime}\right\}$ the $k \times l$ complement of $\boldsymbol{\lambda}$. Equivalently, the Ferrer's diagram of $\boldsymbol{\lambda}^{\prime}$ is the reversed complement in a $k \times \ell$ rectangle of the Ferrer's diagram of $\boldsymbol{\lambda}$. Also, observe that

$$
N\left(\boldsymbol{\lambda}^{\prime}\right)=k \ell-N(\boldsymbol{\lambda})
$$

Proposition 3.3. Bounded partitions $\boldsymbol{\lambda}, \boldsymbol{\mu} \in \mathrm{B}_{k, \ell}$ are complementary partitions, $\boldsymbol{\mu}=\boldsymbol{\lambda}^{\prime}$, if and only if $\boldsymbol{\lambda}^{+}=\boldsymbol{\mu}^{-}$.

We now come to the main result of this section.

Theorem 3.4. Bounded partitions $\boldsymbol{\lambda} \in \mathrm{B}_{k, \ell}$ and $\hat{\boldsymbol{\lambda}} \in \mathrm{B}_{\ell, k}$ are conjugate partitions if and only if $\boldsymbol{\lambda}^{+}$and $\hat{\boldsymbol{\lambda}}^{-}$are complementary subsets of $\{0,1, \ldots, n\}$.

\subsection{Row reduced echelon form}

Let $\boldsymbol{\lambda} \in \mathrm{B}_{k, \ell}$ be a bounded partition. We will say that a $k \times \ell$ matrix $\left\{a_{i j}\right\}$ where $1 \leq i \leq k$ and $1 \leq j \leq \ell$ is $\lambda$-bounded if $a_{i j}=0$ for all $j \leq \ell-\lambda_{i}$.

Next we describe a useful bijection between the set of $\boldsymbol{\lambda}$-bounded matrices and matrices in reduced row echelon form (RREF). Let $\mathrm{R}_{k, \ell}$ denote the set of $k \times(k+\ell)$ matrices that have rank $k$ and that are in RREF. When dealing with matrices in $\mathrm{R}_{k, \ell}$ we adopt the convention that $n=$ $k+\ell-1$, while $1 \leq i \leq k$ and $0 \leq j \leq n$ serve as the row and column indices, respectively. These index conventions are convenient because of subsequent applications to polynomial subspaces; the column indices $\{0,1, \ldots, n\}$ enumerate the possible degrees of a polynomial in $\mathcal{P}_{n}$.

Every matrix in $\mathrm{R}_{k, \ell}$ can be uniquely block-partitioned as follows

$$
\left(\begin{array}{ccccccc}
\mathrm{I}_{1} & B_{11} & 0 & B_{12} & \cdots & 0 & B_{1 r} \\
0 & 0 & \mathrm{I}_{2} & B_{22} & \cdots & 0 & B_{2 r} \\
\vdots & \vdots & \vdots & \vdots & \ddots & \vdots & \vdots \\
0 & 0 & 0 & 0 & \cdots & \mathrm{I}_{r} & B_{r r}
\end{array}\right),
$$

where the submatrices $B_{p q}, 1 \leq p \leq q \leq r$ are blocks of size $k_{p} \times \ell_{q}$ and the blocks $\mathrm{I}_{p}$ denote a $k_{p} \times k_{p}$ identity matrix. Here

$$
k_{1}+\cdots+k_{r}=k, \quad \ell_{1}+\cdots+\ell_{r}=\ell,
$$

\footnotetext{
${ }^{4}$ When $n=-1$ we are speaking of subsets of the empty set. When $n=0$ we are referring to subsets of $\{0\}$.
} 
with $k_{1}, \ell_{r} \geq 0$ and all other $k_{i}, \ell_{j}$ strictly positive. The possibilities $k_{1}=0$ and $\ell_{r}=0$ correspond to 3 degenerate subcases, shown below:

$$
\begin{aligned}
& \left(\begin{array}{cccccc}
0 & \mathrm{I}_{2} & B_{22} & \cdots & 0 & B_{2 r} \\
\vdots & \vdots & \vdots & \ddots & \vdots & \vdots \\
0 & 0 & 0 & \cdots & \mathrm{I}_{r} & B_{r r}
\end{array}\right), \quad k_{1}=0, \quad \ell_{r}>0 \\
& \left(\begin{array}{cccccc}
\mathrm{I}_{1} & B_{11} & 0 & B_{12} & \cdots & 0 \\
0 & 0 & \mathrm{I}_{2} & B_{22} & \cdots & 0 \\
\vdots & \vdots & \vdots & \vdots & \ddots & \vdots \\
0 & 0 & 0 & 0 & \cdots & \mathrm{I}_{r}
\end{array}\right), \quad k_{1}>0, \quad \ell_{r}=0 \\
& \left(\begin{array}{ccccc}
0 & \mathrm{I}_{2} & B_{22} & \cdots & 0 \\
\vdots & \vdots & \vdots & \ddots & \vdots \\
0 & 0 & 0 & \cdots & \mathrm{I}_{r}
\end{array}\right), \quad k_{1}=\ell_{r}=0 .
\end{aligned}
$$

Also note that $\mathrm{R}_{k, 0}$ is a singleton set consisting of the $k \times k$ identity matrix.

Deleting the pivot columns from a matrix in RREF results in a $\boldsymbol{\lambda}$-bounded matrix for a certain bounded partition $\boldsymbol{\lambda} \in \mathrm{B}_{k, \ell}$.

Proposition 3.5. Let $\boldsymbol{\lambda} \in \mathrm{B}_{k, \ell}$ be a bounded partition. Let $\boldsymbol{\nu}=\boldsymbol{\lambda}^{+}$and let $\boldsymbol{\mu}$ be the increasing enumeration of the complement $\{0,1, \ldots, n\} \backslash\left\{\nu_{i}\right\}$. Let $\left\{a_{i j}\right\}$ be a $\boldsymbol{\lambda}$-bounded matrix, and let $\boldsymbol{A}=\left(A_{i j}\right)$ be the $k \times(k+\ell)$ matrix defined by

$$
\begin{aligned}
& A_{i \mu_{j}}=a_{i j}, \quad i=1, \ldots, k, \quad j=1, \ldots, \lambda_{i}, \\
& A_{i \nu_{i}}=1,
\end{aligned}
$$

with all other entries zero. Then, $A$ is in RREF; $\left\{\nu_{i}\right\}$ is the enumeration of the pivot columns of $A$; and $\left\{\mu_{j}\right\}$ is the enumeration of the non-pivot columns. Conversely, every $A \in \mathrm{R}_{k, \ell}$ admits a unique such representation in terms of a $\boldsymbol{\lambda}$-bounded matrix for some $\boldsymbol{\lambda} \in \mathrm{B}_{k, \ell}$.

The above construction associates the trivial partition to the most extreme form of (3.6), the matrix composed of $\ell$ zero columns followed by the $k \times k$ identity matrix.

\subsection{The shape of a polynomial subspace}

Next, we apply the above results on reduced row echelon form to polynomial subspaces. Fix integers $n \geq 0$ and $1 \leq k \leq n+1$ and set $\ell=n+1-k$. For a non-zero polynomial $p \in \mathcal{P}_{n}$ and $b \in \mathbb{C}$ we define

$$
\operatorname{ord}_{b} p=\min \left\{j \geq 0: p^{(j)}(b) \neq 0\right\} .
$$

As per the usual convention, $\operatorname{ord}_{b} 0=\infty$. For a polynomial subspace $U$, we define

$$
\operatorname{ord}_{b} U=\min \left\{\operatorname{ord}_{b} p: p \in U\right\} .
$$

Equivalently, if $U$ is non-trivial, $\nu=\operatorname{ord}_{b} U$ is the largest integer such that $(z-b)^{\nu}$ divides all $p(z) \in U$.

Proposition 3.6. Let $U$ be a polynomial subspace and let $b \in \mathbb{C}$. Then, the subspace

$$
U^{\prime}=\left\{p \in U: \operatorname{ord}_{b} p>\operatorname{ord}_{b} U\right\}
$$

has codimension 1 in $U$. 
For $U \in \mathcal{G}_{k} \mathcal{P}_{n}$ and $b \in \mathbb{C}$, we inductively define a flag of subspaces

$$
U=U_{1} \supset U_{2} \supset \cdots \supset U_{k}, \quad \operatorname{dim} U_{i}=k+1-i,
$$

by setting $U_{1}=U$ and

$$
U_{i+1}=U_{i}^{\prime}, \quad i=1, \ldots, k-1 .
$$

Set

$$
\nu_{i}=\operatorname{ord}_{b} U_{i}, \quad \lambda_{i}=n-\nu_{i}+i-k, \quad i=1, \ldots, k .
$$

Observe that $\boldsymbol{\nu} \in \mathrm{P}_{k, \ell}$, that $\boldsymbol{\lambda} \in \mathrm{B}_{k, \ell}$ and that $\boldsymbol{\nu}=\boldsymbol{\lambda}^{+}$, c.f. (3.1). For reasons explained below, we will call $\boldsymbol{\nu}$ the b-pivot sequence and call $\boldsymbol{\lambda}$ the b-shape of $U$.

For polynomials $p_{1}, \ldots, p_{k}$, let

$$
\mathrm{M}_{n}\left(p_{1}, \ldots, p_{k}\right)=\left(\begin{array}{cccc}
p_{1} & p_{1}^{\prime} & \cdots & p_{1}^{(n)} \\
p_{2} & p_{2}^{\prime} & \cdots & p_{2}^{(n)} \\
\vdots & \vdots & \ddots & \vdots \\
p_{k} & p_{k}^{\prime} & \cdots & p_{k}^{(n)}
\end{array}\right)
$$

denote the $k \times(n+1)$ matrix of indicated derivatives. For $U \in \mathcal{G}_{k} \mathcal{P}_{n}$ and $b \in \mathbb{C}$, let $\left\{U_{i}\right\}$ be the $\operatorname{ord}_{b}$ filtration defined by (3.7).

Proposition 3.7. There exists a unique basis $p_{1}, \ldots, p_{k}$ of $U$ such that $p_{i} \in U_{i}$ and such that

$$
p_{i}^{\left(\nu_{i}\right)}(b)=1, \quad p_{i}^{\left(\nu_{j}\right)}(b)=0, \quad j>i .
$$

This basis is characterized by the property that $\mathrm{M}_{n}\left(p_{1}, \ldots, p_{k}\right)(b)$ is in RREF.

Definition 3.8. Henceforth, we will refer to the above $p_{1}, \ldots, p_{k}$ as the b-reduced basis of $U$ and set

$$
\mathrm{M}(U, b)=\mathrm{M}_{n}\left(p_{1}, \ldots, p_{k}\right)(b) \in \mathrm{R}_{k, \ell} .
$$

The sequence $\left\{\nu_{i}\right\}$ enumerates the pivot columns of $\mathrm{M}(U, b)$. Also observe that if we delete the pivot columns from $\mathrm{M}(U, b)$, we obtain a $\boldsymbol{\lambda}$-bounded matrix.

Proposition 3.9. Fix $b \in \mathbb{C}$. Let $\boldsymbol{\lambda}$ be the b-shape of $U$, and $p_{1}, \ldots, p_{k}$ the b-reduced basis. Then,

$$
p_{i}(z)=\frac{(z-b)^{\nu_{i}}}{\nu_{i} !}+\sum_{j=1}^{\ell} a_{i j} \frac{(z-b)^{\mu_{j}}}{\mu_{j} !}, \quad i=1, \ldots, k .
$$

where $\boldsymbol{\nu}=\boldsymbol{\lambda}^{+}, \boldsymbol{\mu}=\boldsymbol{\lambda}^{*-}$, and where the matrix

$$
a_{i j}=p_{i}^{\left(\mu_{j}\right)}(b), \quad i=1, \ldots, k, \quad j=1, \ldots, \ell,
$$

is $\boldsymbol{\lambda}$-bounded.

Proof. By Theorem 3.4, $\boldsymbol{\mu}$ enumerates the non-pivot columns of $M(U, b)$. Relation (3.9) follows by (3.10) and by Proposition 3.7. Observe that, by (3.1)

$$
\begin{aligned}
\lambda_{i} & =n-\nu_{i}-(k-i)=\#\left(\text { columns to the right of } \nu_{i}\right)-\#\left(\text { pivots to the right of } \nu_{i}\right) \\
& =\#\left(\text { non-pivots to the right of } \nu_{i}\right)
\end{aligned}
$$


More formally,

$$
\lambda_{i}=\#\left\{j: \mu_{j}>\nu_{i}\right\} .
$$

Hence $\mu_{j} \leq \nu_{i}$ if and only if $j \leq \ell-\lambda_{i}$. However, $\mu_{j}$ cannot possibly equal $\nu_{i}$. Hence, if $j \leq \ell-\lambda_{i}$, then $\mu_{j}<\nu_{i}=\operatorname{ord}_{b} p_{i}$, and therefore, $a_{i j}=p_{i}^{\left(\mu_{j}\right)}(b)=0$.

Theorem 3.10. Let $U \in \mathcal{G}_{k} \mathcal{P}_{n}$ and $U^{*} \in \mathcal{G}_{\ell} \mathcal{P}_{n}$ be dual polynomial subspaces. Fix $b \in \mathbb{C}$ and let $\boldsymbol{\lambda}$ be the b-shape of $U$. Then, the conjugate partition $\boldsymbol{\lambda}^{*}$ is the b-shape of the apolar dual $U^{*}$. Furthermore, with $\boldsymbol{\nu}, \boldsymbol{\mu},\left\{a_{i j}\right\}$ as above, and with $\boldsymbol{\nu}^{*}=\boldsymbol{\lambda}^{*+}, \boldsymbol{\mu}^{*}=\boldsymbol{\lambda}^{-}$and

$$
a_{k+1-i, \ell+1-j}^{*}=(-1)^{\nu_{i}+\mu_{j}+1} a_{i j}
$$

the reduced basis of $U^{*}$ is given by

$$
p_{j}^{*}(z)=\frac{(z-b)^{\nu_{j}^{*}}}{\nu_{j}^{*} !}+\sum_{i=1}^{k} a_{i j}^{*} \frac{(z-b)^{\mu_{i}^{*}}}{\mu_{i}^{*} !}, \quad j=1, \ldots, \ell .
$$

Proof. Since the bilinear form $\gamma$ described in (2.1) is $\mathrm{SL}_{2} \mathbb{C}$ invariant, we also have

$$
n ! \gamma\left(\frac{(z-b)^{j}}{j !}, \frac{(z-b)^{k}}{k !}\right)= \begin{cases}(-1)^{j}, & \text { if } j+k=n, \\ 0, & \text { otherwise. }\end{cases}
$$

Recall that

$$
\nu_{i}+\mu_{k+1-i}^{*}=n, \quad \mu_{j}+\nu_{\ell+1-j}^{*}=n .
$$

Also, by Theorem 3.4, $\left\{\nu_{j}^{*}\right\}$ and $\left\{\mu_{i}^{*}\right\}$ are complementary enumerations of the set $\{0,1, \ldots, n\}$. Hence for fixed $i, j$ we have, by the definitions (3.9), (3.12) and by (3.13),

$$
\gamma\left(p_{i}, p_{\ell+1-j}^{*}\right)=(-1)^{\nu_{i}} a_{k+1-i, \ell+1-j}^{*}+(-1)^{\mu_{j}} a_{i, j}=0 .
$$

The above discussion, based on a finite parameter $b \in \mathbb{C}$, has an analogous development in terms of $\infty$. Fix $k, \ell, n$ as above. For a given polynomial $p \in \mathcal{P}_{n}$, we call $\infty$ a root of multiplicity $\nu$ if $\operatorname{deg} p=n-\nu$. In this sense,

$$
\operatorname{ord}_{\infty} p=n-\operatorname{deg} p .
$$

Let us also define

$$
\operatorname{deg} U=\max \{\operatorname{deg} p: p \in U\} .
$$

Equivalently, if $U$ is non-trivial, then $m=\operatorname{deg} U$ is the smallest integer such that $U \subset \mathcal{P}_{m}$. We come to the following analogue of Proposition 3.6.

Proposition 3.11. Let $U$ be a finite-dimensional polynomial subspace. Then, the subspace

$$
U^{\prime}=\{p \in U: \operatorname{deg} p<\operatorname{deg} U\}
$$

has codimension 1 in $U$.

For $U \in \mathcal{G}_{k} \mathcal{P}_{n}$, we define a flag of subspaces $\hat{U}_{k} \subset \cdots \subset \hat{U}_{1}$ by setting $\hat{U}_{1}=U$ and

$$
\hat{U}_{i+1}=\hat{U}_{i}^{\prime}, \quad i=1, \ldots, k-1 .
$$

We define the the $\infty$-shape and the $\infty$-pivot sequence as follows:

$$
\begin{aligned}
& \hat{\lambda}_{i}=\operatorname{deg} \hat{U}_{i}-k+i, \quad i=1, \ldots, k, \\
& \hat{\nu}_{i}=\operatorname{deg} \hat{U}_{k+1-i} .
\end{aligned}
$$

Observe that $\hat{\boldsymbol{\nu}}=\hat{\boldsymbol{\lambda}}^{-}$. 
Proposition 3.12. There exists a unique basis $p_{1}, \ldots, p_{k} \in U$ such that each $p_{i} \in \hat{U}_{k+1-i}$ and such that

$$
p_{i}^{\left(\hat{\nu}_{i}\right)}(0)=1, \quad p_{i}^{\left(\hat{\nu}_{j}\right)}(0)=0, \quad j<i
$$

We will refer to such a basis as the $\infty$-reduced basis of $U$. We can now state the following analogue of Theorem 3.10.

Theorem 3.13. Let $U \in \mathcal{G}_{k} \mathcal{P}_{n}$ and $U^{*} \in \mathcal{G}_{\ell} \mathcal{P}_{n}$ be dual polynomial subspaces. Then $\hat{\boldsymbol{\lambda}}$ and $\hat{\boldsymbol{\lambda}}^{*}$, the respective $\infty$-shapes, are conjugate partitions. The respective $\infty$-reduced bases are given by

$$
\begin{aligned}
& p_{i}(z)=\frac{z^{\hat{\nu}_{i}}}{\hat{\nu}_{i} !}+\sum_{j=1}^{\ell} a_{i j} \frac{z^{\hat{\mu}_{j}}}{\hat{\mu}_{j} !}, \quad i=1, \ldots, k, \quad \hat{\boldsymbol{\nu}}=\hat{\boldsymbol{\lambda}}^{-}, \quad \hat{\boldsymbol{\mu}}=\hat{\boldsymbol{\lambda}}^{*+}, \\
& p_{j}^{*}(z)=\frac{z^{\hat{\nu}_{j}^{*}}}{\hat{\nu}_{j}^{* !} !}+\sum_{i=1}^{k} a_{i j}^{*} \frac{z^{\hat{\mu}_{i}^{*}}}{\hat{\mu}_{i}^{*} !}, \quad j=1, \ldots, \ell, \quad \hat{\boldsymbol{\nu}}^{*}=\hat{\boldsymbol{\lambda}}^{*-}, \quad \hat{\boldsymbol{\mu}}^{*}=\hat{\boldsymbol{\lambda}}^{+},
\end{aligned}
$$

where $a_{i j}$ and $a_{i j}^{*}$ are related by (3.11).

Finally, we combine order and degree reductions to obtain a useful characterization of subspaces generated by monomials. Let $U \in \mathcal{G}_{k} \mathcal{P}_{n}$ be a polynomial subspace. Let $\nu_{1}<\cdots<\nu_{k}$ be the 0 -pivot sequence and let $\hat{\nu}_{1}<\cdots<\hat{\nu}_{k}$ be the $\infty$-pivot sequence. These correspond to the following bases of $U$ :

$$
\begin{aligned}
& p_{i}(z)=z^{\nu_{i}} / \nu_{i} !+\text { higher order terms, } \\
& \hat{p}_{i}(z)=z^{\hat{\nu}_{i}} / \hat{\nu}_{i} !+\text { lower degree terms. }
\end{aligned}
$$

Proposition 3.14. For $j=1, \ldots, k$, we have $\nu_{j} \leq \hat{\nu}_{j}$. If $\nu_{j}=\hat{\nu}_{j}$ for a particular $j$, then the monomial $z^{j} \in U$.

Proof. For each $j=1, \ldots, k$ choose a $p_{j} \in U$ such that $\operatorname{ord}_{0} p_{j}=\nu_{j}$ and such that $\pi_{j}:=\operatorname{deg} p_{j}$ is as small as possible. Observe that for $i<j$ we must have $\pi_{i} \neq \pi_{j}$; otherwise a linear combination of $p_{i}$ and $p_{j}$ would have the same order as $p_{i}$ but a smaller degree. It follows that $\pi_{1}, \ldots, \pi_{k}$ enumerates the degree pivot set $\left\{\hat{\nu}_{j}\right\}$, although not necessarily in any particular order. Observe that for every polynomial $p$, we have $\operatorname{ord}_{0} p \leq \operatorname{deg} p$ with equality if and only if $p$ is a monomial. Hence, $\nu_{j} \leq \pi_{j}$ with equality if and only if $p_{j}$ is a monomial. Hence, for $i \leq j$ we have

$$
\nu_{i} \leq \nu_{j} \leq \pi_{j}
$$

Since $\left\{\pi_{i}, \ldots, \pi_{k}\right\}$ is a set with $k+1-i$ distinct elements, by the pigeonhole principle,

$$
\nu_{i} \leq \min \left\{\pi_{i}, \ldots, \pi_{k}\right\} \leq \tilde{\nu}_{i}, \quad i=1, \ldots, k
$$

As for the final assertion, observe that if $i<j$, then $\nu_{i}<\pi_{j}$. Hence, if $\nu_{i}=\hat{\nu}_{i}$, then necessarily $\pi_{i}=\nu_{i}$; this means that $p_{i}$ is a monomial.

Corollary 3.15. A polynomial subspace $U \in \mathcal{G}_{k} \mathcal{P}_{n}$ is spanned by monomials if and only if the order pivots match the degree pivots: $\nu_{i}=\hat{\nu}_{i}$ for all $i=1, \ldots, k$. 


\section{The Wronskian covariant}

In this section we introduce the Wronskian of a polynomial subspace, a key covariant that will allow us to solve the equivalence problem. Throughout, integers $k, \ell, n$ are as in the preceding section. For an ordered set of polynomials $p_{1}, \ldots, p_{k} \in \mathcal{P}_{n}$, we define their Wronskian to be the polynomial

$$
\mathrm{W}\left(p_{1}, \ldots, p_{k}\right)=\operatorname{det} \mathrm{M}_{k-1}\left(p_{1}, \ldots, p_{k}\right)
$$

see (3.8) for the definition of M. For an integer sequence $\mu_{1}, \ldots, \mu_{k}$ define

$$
\mathrm{V}(\boldsymbol{\mu})=\mathrm{V}\left(\mu_{1}, \ldots, \mu_{k}\right)=\prod_{1 \leq i<j \leq k}\left(\mu_{j}-\mu_{i}\right)
$$

Observe that if $\boldsymbol{\mu}$ is strictly increasing, then $V(\boldsymbol{\mu})>0$.

Proposition 4.1. For a bounded partition $\boldsymbol{\lambda} \in \mathrm{B}_{k, \ell}$ and $\boldsymbol{\nu}=\boldsymbol{\lambda}^{-}$we have

$$
\mathrm{W}\left(z^{\nu_{1}}, \ldots, z^{\nu_{k}}\right)=\mathrm{V}(\boldsymbol{\nu}) z^{N(\boldsymbol{\lambda})} .
$$

Proof. By definition,

$$
\mathrm{W}\left(z^{\nu_{1}}, \ldots, z^{\nu_{k}}\right)=\sum_{\pi} \prod_{i=1}^{k}\left(\nu_{\pi_{i}}\right)_{i-1} z^{\nu_{\pi_{i}}-i+1},
$$

where

$$
(x)_{j}=x(x-1) \cdots(x-j+1)
$$

is the usual falling factorial, and where $\pi$ ranges over all permutations of $\{1, \ldots, k\}$. By (3.2),

$$
\sum_{i=1}^{k}\left(\nu_{\pi_{i}}-i+1\right)=\sum_{i=1}^{k}\left(\nu_{i}-i+1\right)=\sum_{i=1}^{k} \lambda_{k+1-i}=N(\boldsymbol{\lambda}) .
$$

Hence, by inspection,

$$
\mathrm{W}\left(z^{\nu_{1}}, \ldots, z^{\nu_{k}}\right)=P\left(\nu_{1}, \ldots, \nu_{k}\right) z^{N}
$$

where $P$ is a polynomial of degree $k$ in each $\nu_{i}$. Since the Wronskian is an alternating mapping, the function $P$ must be an alternating polynomial. Hence, $\nu_{i}-\nu_{j}$ is a factor of $P$ for all $i \neq j$, and hence, $P$ and $\mathrm{V}\left(\nu_{1}, \ldots, \nu_{k}\right)$ agree up to a constant factor. By comparing the highest order terms of $\nu_{k}$ in both $\mathrm{V}\left(\nu_{1}, \ldots, \nu_{k}\right)$ and (4.1) we see that this factor is 1.

Proposition 4.2. Let $p_{1}, \ldots, p_{k} \in \mathcal{P}_{n}$ be linearly independent polynomials, and let $\hat{\boldsymbol{\lambda}}$ be the $\infty$-shape of the subspace $U=\operatorname{span}\left\{p_{1}, \ldots, p_{k}\right\}$ as defined in (3.15). Then,

$$
\operatorname{deg} \mathrm{W}\left(p_{1}, \ldots, p_{k}\right)=\sum_{i} \hat{\lambda}_{i} \leq k \ell
$$

Equality holds if and only if $p_{1}, \ldots, p_{k}$ are linearly independent modulo $\mathcal{P}_{\ell-1}$. 
Proof. It suffices to establish (4.2) in the case where $p_{1}, \ldots, p_{k}$ is the $\infty$-reduced basis. Let $\hat{\nu}_{i}=$ $\operatorname{deg} p_{k+1-i}$ be the pivot sequence. By the multi-linearity of the Wronskian and by Proposition 4.1,

$$
\mathrm{W}\left(p_{k}, \ldots, p_{1}\right)=\frac{\mathrm{V}\left(\hat{\nu}_{1}, \ldots, \hat{\nu}_{k}\right)}{\hat{\nu}_{1} ! \cdots \hat{\nu}_{k} !} z^{N(\hat{\boldsymbol{\lambda}})}+\text { lower degree terms. }
$$

This establishes the equality in (4.2).

We now prove the second assertion. Since $\hat{\boldsymbol{\lambda}}$ is a $k \times \ell$ bounded partition, we have $N(\hat{\boldsymbol{\lambda}}) \leq k \ell$ with equality if and only if $\hat{\boldsymbol{\lambda}}$ is rectangular. By (3.16) and (3.2), this is true if and only if $\hat{\nu}_{1}=\hat{\lambda}_{k}=\ell$. Since $\hat{\nu}_{1}<\cdots<\hat{\nu}_{k} \leq n$ and since $\ell+k-1=n$, the partition $\hat{\lambda}$ is rectangular if and only if $p_{1}, \ldots, p_{k}$ are linearly independent modulo $\mathcal{P}_{\ell-1}$.

Let $U \in \mathcal{G}_{k} \mathcal{P}_{n}$ be a polynomial subspace and let $p_{1}, \ldots, p_{k} \in U$ be a basis. In light of Proposition 4.2 , we may regard the Wronskian $\mathrm{W}\left(p_{1}, \ldots, p_{k}\right)$ as an element of $\mathcal{P}_{k \ell}$. Since $\mathrm{W}$ is a multi-linear, alternating mapping,

$$
p_{1} \wedge \cdots \wedge p_{k} \mapsto \mathrm{W}\left(p_{1}, \ldots, p_{k}\right), \quad p_{1}, \ldots, p_{k} \in \mathcal{P}_{n}
$$

defines a linear transformation $\mathrm{W}: \Lambda^{k} \mathcal{P}_{n} \rightarrow \mathcal{P}_{k \ell}$; here $\Lambda^{k}$ denotes the $k^{\text {th }}$ exterior product of a vector space.

Let us give a basis description of this linear transformation. For a sequence of integers $0 \leq \nu_{1}, \ldots, \nu_{k} \leq n$ define

$$
z^{\nu}=z^{\nu_{1}} \wedge \cdots \wedge z^{\nu_{k}} \in \Lambda^{k} \mathcal{P}_{n}
$$

In light of the bijective correspondence $\mathrm{B}_{k, \ell} \rightarrow \mathrm{P}_{k, \ell}$ defined in Section 3.1, the set $\left\{z^{\boldsymbol{\lambda}^{-}}: \boldsymbol{\lambda} \in\right.$ $\left.\mathrm{B}_{k, \ell}\right\}$ is a basis of $\Lambda^{k} \mathcal{P}_{n}$. We can therefore describe $\mathrm{W}$ by stipulating

$$
\mathrm{W}\left(z^{\boldsymbol{\lambda}^{-}}\right)=\mathrm{V}\left(\boldsymbol{\lambda}^{-}\right) z^{N(\boldsymbol{\lambda})}, \quad \boldsymbol{\lambda} \in \mathrm{B}_{k, \ell} .
$$

Theorem 4.3. The linear transformation $\mathrm{W}: \Lambda^{k} \mathcal{P}_{n} \rightarrow \mathcal{P}_{k \ell}$ is $\mathrm{SL}_{2} \mathbb{C}$-equivariant.

Proof. Let

$$
z=\frac{a \hat{z}+b}{c \hat{z}+d}, \quad a d-b c=1
$$

be a fractional linear transformation, let $p_{1}, \ldots, p_{k} \in \mathcal{P}_{n}$, and let

$$
\hat{p}_{i}(\hat{z})=(c \hat{z}+d)^{n} p_{i}(z), \quad i=1, \ldots, k
$$

be the transformed polynomials. The claim is that

$$
\mathrm{W}\left(\hat{p}_{1}, \ldots, \hat{p}_{k}\right)(\hat{z})=(c \hat{z}+d)^{k \ell} \mathrm{W}\left(p_{1}, \ldots, p_{k}\right)(z) .
$$

It suffices to consider 3 kinds of transformation: translations, homotheties, and inversions. Consider a translation transformation

$$
\hat{p}(\hat{z})=p(z), \quad p \in \mathcal{P}_{n}, \quad z=\hat{z}+b, \quad b \in \mathbb{C} .
$$

Here $a=1, c=0, d=1$. Observe that $\hat{p}^{(j)}(\hat{z})=p^{(j)}(z)$. Equation (4.4) follows immediately.

Next, consider a homothety transformation

$$
\hat{p}(\hat{z})=a^{-n} p(z), \quad z=a^{2} \hat{z}, \quad a \neq 0
$$


This corresponds to $b=c=0$ and $d=1 / a$ in (4.3). Observe that

$$
\hat{p}^{(j)}(\hat{z})=a^{2 j-n} p^{(j)}(z) .
$$

Hence,

$$
\mathrm{W}\left(\hat{p}_{1}, \ldots, \hat{p}_{k}\right)(\hat{z})=a^{k(k-1)-n k} \mathrm{~W}\left(p_{1}, \ldots, p_{k}\right)(z)=a^{-k \ell} \mathrm{W}\left(p_{1}, \ldots, p_{k}\right)(z),
$$

in full accordance with (4.4).

Finally, consider an inversion

$$
\hat{p}(\hat{z})=\hat{z}^{n} p(z), \quad z=-1 / \hat{z} .
$$

Let $R_{i}(z), i=0,1, \ldots, k-1$ denote the $i^{\text {th }}$ column vector $\left(p_{1}^{(i)}(z), \ldots, p_{k}^{(i)}(z)\right)^{t}$ of $\mathrm{M}_{k-1}\left(p_{1}\right.$, $\left.\ldots, p_{k}\right)$, and let $\hat{R}_{i}(\hat{z})$ be the corresponding column vector formed from the derivatives of $\hat{p}_{i}(\hat{z})$. Observe that

$$
\hat{p}^{\prime}(\hat{z})=\hat{z}^{n-2} p^{\prime}(z)+n \hat{z}^{n-1} p(z),
$$

whence

$$
\hat{R}_{1}(\hat{z})=\hat{z}^{n-2} R_{1}(z)+n \hat{z}^{n-1} R_{0}(z) .
$$

More generally,

$$
\hat{R}_{i}(\hat{z})=\hat{z}^{n-2 i} R_{i}(z)+\cdots, \quad i=0, \ldots, k-1,
$$

where the remainder is a linear combination of $R_{0}(z), \ldots, R_{i-1}(z)$. Since

$$
\sum_{i=0}^{k-1} n-2 i=n k-k(k-1)=k \ell,
$$

by the multi-linearity and skew-symmetry of the determinant it follows that

$$
\mathrm{W}\left(\hat{p}_{1}, \ldots, \hat{p}_{k}\right)(\hat{z})=\operatorname{det}\left(\hat{R}_{0}(\hat{z}), \ldots, \hat{R}_{k-1}(\hat{z})\right)=\hat{z}^{k \ell} \mathrm{W}\left(p_{1}, \ldots, p_{k}\right)(z),
$$

as was to be shown.

Definition 4.4. By a slight abuse of notation we also use $\mathrm{W}$ to denote the corresponding Wronski mapping $\mathrm{W}: \mathcal{G}_{k} \mathcal{P}_{n} \rightarrow \mathcal{G}_{1} \mathcal{P}_{k \ell}$, defined by

$$
\mathrm{W}(U)=\operatorname{span} \mathrm{W}\left(p_{1}, \ldots, p_{k}\right), \quad U \in \mathcal{G}_{k} \mathcal{P}_{n},
$$

where $p_{1}, \ldots, p_{k} \in U$ is any basis. We refer to $\mathrm{W}(U)$ as the Wronskian covariant of the polynomial subspace $U$.

Being an element of $\mathcal{G}_{1} \mathcal{P}_{k \ell}$, we may regard $\mathrm{W}(U)$ as a polynomial of degree $\leq k \ell$ defined up to a non-zero scalar multiple. However, the $k \ell$ roots of $\mathrm{W}(U)$ are defined unambiguously. These roots transform covariantly with respect to projective transformations.

The relationship of the linear and non-linear Wronski maps is conveniently summarized by the following diagram; the top arrow is the Plücker embedding, the right arrow is the projectivization of the linear Wronski map and the left diagonal arrow is the non-linear Wronski map

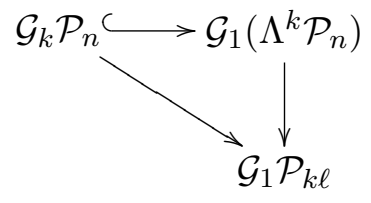

We can now formulate the relationship between roots of the Wronskian and the possible shapes of a subspace. Throughout, recall that the order of a polynomial at $\infty$ is defined by (3.14). 
Theorem 4.5. Let $U \in \mathcal{G}_{k} \mathcal{P}_{n}$ be a subspace and let $b \in \mathbb{C} \cup\{\infty\}$ be given. We have

$$
\operatorname{ord}_{b} \mathrm{~W}(U)=N\left(\boldsymbol{\lambda}^{\prime}\right)=k \ell-N(\boldsymbol{\lambda}),
$$

where $\boldsymbol{\lambda}$ is the $b$-shape of $U$ and $\boldsymbol{\lambda}^{\prime}$ is the complementary partition.

Proof. This follows from Proposition 4.2, which treats the $b=\infty$ case. By Proposition 4.2,

$$
\operatorname{ord}_{\infty} \mathrm{W}(U)=k \ell-N(\boldsymbol{\lambda})
$$

where $\boldsymbol{\lambda}$ is the $\infty$-shape of $U$. The present assertion now follows by the equivariance of $\mathrm{W}$.

The description of simple roots deserves explicit mention.

Corollary 4.6. Let $U \in \mathcal{G}_{k} \mathcal{P}_{n}$ be a subspace. Fix $b \in \mathbb{C}$ and let $\boldsymbol{\lambda}$ be the b-shape of $U$ and $\boldsymbol{\nu}=\boldsymbol{\lambda}^{+}$ the pivot sequence. Then, $b$ is a simple root of $\mathrm{W}(U)$ if and only if $\nu_{j}=j-1, j=1, \ldots, k-1$ and $\nu_{k}=k$. Equivalently, $b$ is a simple root if and only if $\mathrm{M}(U, b)$ has the form

$$
\mathrm{M}(U, b)=\left(\begin{array}{cccc}
I_{1} & B_{11} & 0 & B_{12} \\
0 & 0 & 1 & B_{22}
\end{array}\right)
$$

with $B_{11}, B_{12}, B_{22}$ having dimensions $(k-1) \times 1,(k-1) \times(\ell-1)$ and $1 \times(\ell-1)$, respectively; c.f. (3.3).

We also have the following key result asserting the invariance of the Wronskian with respect to apolar duality.

Theorem 4.7. A polynomial subspace $U \in \mathcal{G}_{k} \mathcal{P}_{n}$ and its apolar dual $U^{*} \in \mathcal{G}_{\ell} \mathcal{P}_{n}$ have the same Wronskian covariant; $\mathrm{W}(U)=\mathrm{W}\left(U^{*}\right)$.

Proof. Theorems 3.10 and 3.13 establish shape duality of $U$ and $U^{*}$. By Proposition 3.2, we have $N(\boldsymbol{\lambda})=N\left(\boldsymbol{\lambda}^{*}\right)$. Therefore, by Theorem 4.5 the Wronskians $\mathrm{W}(U)$ and $\mathrm{W}\left(U^{*}\right)$ have the same roots with the same multiplicities.

\subsection{Additional properties of the Wronski map}

Call a multi-vector in $\Lambda^{k} \mathcal{P}_{n}$ decomposable if it can be given as $p_{1} \wedge \cdots \wedge p_{k}$ for some linearly independent $p_{1}, \ldots, p_{k} \in \mathcal{P}_{n}$. A basis of a $k$-dimensional subspace $U \in \mathcal{G}_{k} \mathcal{P}_{n}$ defines a decomposable multi-vector, unique up to scalar multiple. This gives us the Plücker embedding $\mathcal{G}_{k} \mathcal{P}_{n} \hookrightarrow \mathcal{G}_{1} \Lambda^{k} \mathcal{P}_{n}$. Indeed, the Grassmannian $\mathcal{G}_{k} \mathcal{P}_{n}$ can be regarded as a projective variety generated by a collection of quadratic polynomials, the so-called Plücker relations [5, 12].

The domain and codomain of the Wronski mapping $\mathrm{W}: \mathcal{G}_{k} \mathcal{P}_{n} \rightarrow \mathcal{G}_{1} \mathcal{P}_{k \ell}$ are both $k \ell-$ dimensional varieties. This suggests that $W$ has nice properties from the algebro-geometric point of view. The following result is needed in the sequel.

Proposition 4.8. The Wronski map $\mathrm{W}: \mathcal{G}_{k} \mathcal{P}_{n} \rightarrow \mathcal{G}_{1} \mathcal{P}_{k \ell}$ is surjective with finite pre-images of points.

Proof. The kernel of the linear transformation $\mathrm{W}: \Lambda^{k} \mathcal{P}_{n} \rightarrow \mathcal{P}_{k \ell}$ does not contain any primitive multi-vectors; see Proposition 4.1. Hence, the mapping W: $\mathcal{G}_{k} \mathcal{P}_{n} \rightarrow \mathcal{G}_{1} \mathcal{P}_{k \ell}$ is a central projection, and hence is finite and surjective [20, Theorems 4 and 7, Section 5.3]. 
Consideration of the degree of the Wronski map leads to very interesting topics such as the combinatorics of Young tableaux and the Schubert calculus. Indeed, the degree is given by $d(k, l)$ where

$$
d(k, \ell)=\frac{(k-1) ! !(\ell-1) ! !}{n ! !}(k \ell) !, \quad \text { where } \quad j ! !=1 ! 2 ! \cdots j !,
$$

counts the standard Young tableaux of shape $k \times \ell$. See references $[8,12]$ for more details.

Finally, let us mention the following, alternate characterization of the Wronski map [4].

Proposition 4.9. For $p_{1}, \ldots, p_{k} \in \mathcal{P}_{n}$, we have

$$
\begin{aligned}
& W\left(p_{1}, \ldots, p_{k}\right)(z)\left(w^{0} \wedge w^{1} \wedge \cdots \wedge w^{n}\right) \\
& \quad=(k-1) ! ! p_{1}(w) \wedge \cdots \wedge p_{k}(w) \wedge(w-z)^{k} \wedge \cdots \wedge(w-z)^{n} .
\end{aligned}
$$

Proof. Note that

$$
(w-z)^{j}=\sum_{i=0}^{j}\left(\begin{array}{l}
j \\
i
\end{array}\right)(-1)^{i} w^{j-i} z^{i}
$$

is understood to refer to an element of $\Lambda^{1}\left(\mathcal{P}_{n}(w)\right) \otimes \mathcal{P}_{n}(z)$, while relation (4.6) should be understood to hold in $\Lambda^{k}\left(\mathcal{P}_{n}(w)\right) \otimes \mathcal{P}_{n}(z)$. In other words, the wedge product is taken relative to the $w$ variable. For example:

$$
(a w+b z) \wedge(w-z)^{2}=a\left(w^{1} \wedge w^{2}\right)+b z\left(w^{0} \wedge w^{2}\right)-(a+2 b) z^{2}\left(w^{0} \wedge w^{1}\right) .
$$

Relation (4.6) follows from the following observations:

$$
\begin{aligned}
& p_{i}(w)=\sum_{j=0}^{k-1} p_{i}^{(j)}(z) \frac{(w-z)^{j}}{j !}, \quad i=1, \ldots, k \\
& w^{0} \wedge w^{1} \wedge \cdots \wedge w^{n}=(w-z)^{0} \wedge(w-z)^{1} \wedge \cdots \wedge(w-z)^{n} .
\end{aligned}
$$

Formula (4.6) has the following, geometric interpretation. Let us regard $\mathcal{G}_{1} \mathcal{P}_{n}$ as $n$-dimensional projective space and via projectivization identify $k$-dimensional subspaces $U \in \mathcal{G}_{k} \mathcal{P}_{n}$ with $(k-1)$ dimensional flats in $\mathcal{G}_{1} \mathcal{P}_{n}$. For a primitive multivector $p_{1} \wedge \cdots \wedge p_{k}$, let $\left[p_{1} \wedge \cdots \wedge p_{k}\right]$ denote the corresponding element of $\mathcal{G}_{k} \mathcal{P}_{n}$ under the Plücker embedding. The mapping

$$
z \mapsto\left[(w-z)^{n}\right]
$$

describes the rational normal curve in projective space $\mathcal{G}_{1} \mathcal{P}_{n}(w)$. Setting

$$
N(z)=(w-z)^{k} \wedge \cdots \wedge(w-z)^{n} \in \Lambda^{\ell} \mathcal{P}_{n}(w)
$$

the 1-parameter family of corresponding $(\ell-1)$ dimensional osculating flats is described by $[N(z)] \in \mathcal{G}_{\ell} \mathcal{P}_{n}(w)$. Therefore, relation (4.6) implies that the roots of the Wronskian $W(U)(z)$, where $U \in \mathcal{G}_{k} \mathcal{P}_{n}$, correspond to points on the rational normal curve where the $(\ell-1)$ dimensional flat $[N(z)]$ touches the $(k-1)$ dimensional flat $U$ in the ambient $(k+\ell-1)$ dimensional projective space $\mathcal{G}_{1} \mathcal{P}_{n}$. The order of the root corresponds to the dimension of the intersection plus 1 . Finally, note that since the rational normal curve is $\mathrm{SL}_{2} \mathbb{C}$-invariant, the above observations constitute an alternate proof of the equivariance of the Wronski map W. 


\section{The equivalence problem}

\subsection{The generic reduction}

Definition 5.1. Let $U, \hat{U} \in \mathcal{G}_{k} \mathcal{P}_{n}$ be polynomial subspaces. Recall that $U \sim \hat{U}$ means that there exists a transformation $g \in \mathrm{PSL}_{2} \mathbb{C}$ such that $\hat{U}=g \cdot U$. We define $\operatorname{Stab}(U) \subset \mathrm{PSL}_{2} \mathbb{C}$ to be the stabilizer of $U$; that is, the group of transformations $g \in \mathrm{PSL}_{2} \mathbb{C}$ such that $U=g \cdot U$.

In light of Proposition 4.8, every polynomial $Q \in \mathcal{P}_{k \ell}(z)$ corresponds to a finite number of subspaces $U \in \mathcal{G}_{k} \mathcal{P}_{n}$ such that $\mathrm{W}(U)=\operatorname{span} Q$. In particular, if $Q$ has simple roots, then the number of such subspaces, counting multiplicities, is $d(k, \ell)$ as given by $(4.5)^{5}$. The equivariance of the Wronski map, Theorem 4.3, has the following immediate consequences.

Proposition 5.2. For $U \in \mathcal{G}_{k} \mathcal{P}_{n}$ we have $\operatorname{Stab}(U) \subset \operatorname{Stab}(W(U))$.

The above observation leads to an immediate proof of Proposition 1.2. Since a generic polynomial has a trivial symmetry group, the algorithm of [3] reduces the equivalence problem for generic polynomial subspaces to the corresponding equivalence problem for the corresponding Wronskians. Again, it must be emphasized that if $W(U)$ is equivalent to $W(\hat{U})$, where $U, \hat{U} \in$ $\mathcal{G}_{k} \mathcal{P}_{n}$, we cannot conclude that $U \sim \hat{U}$. Rather, in the generic case it suffices to calculate the unique $g \in \mathrm{PSL}_{2}$ such that $W(\hat{U})=g \cdot W(U)$ and then to test whether $\hat{U}=g \cdot U$.

Let us recall the relevant details of the algorithm of $[3,17]$. For $Q \in \mathcal{P}_{N}{ }^{6}$, the following differential operators define a number of key polynomial covariants [17, Chapter 5]:

$$
\begin{aligned}
H= & \frac{1}{2}(Q, Q)^{(2)}=N(N-1)\left[Q Q^{\prime \prime}-\frac{N-1}{N}\left(Q^{\prime}\right)^{2}\right], \\
T= & (Q, H)^{(1)}=-N^{2}(N-1)\left[Q^{2} Q^{\prime \prime \prime}-3 \frac{(N-2)}{N} Q Q^{\prime} Q^{\prime \prime}+2 \frac{(N-1)(N-2)}{N^{2}}\left(Q^{\prime}\right)^{3}\right], \\
V= & Q^{3} Q^{\prime \prime \prime}-4 \frac{(N-3)}{N} Q^{2} Q^{\prime} Q^{\prime \prime}+6 \frac{(N-2)(N-3)}{N^{2}} Q\left(Q^{\prime}\right)^{2} Q^{\prime \prime} \\
& +\frac{(N-1)(N-2)(N-3)}{N^{3}}\left(Q^{\prime}\right)^{4}, \\
U= & (Q, T)^{(1)}=N^{3}(N-1) V-3 \frac{(N-2)}{(N-1)} H^{2} .
\end{aligned}
$$

Above, for polynomials $Q \in \mathcal{P}_{n}$ and $R \in \mathcal{P}_{m}$ the expression

$$
(Q, R)^{(r)}(z)=r ! \sum_{k=0}^{r}(-1)^{k}\left(\begin{array}{c}
n-r+k \\
k
\end{array}\right)\left(\begin{array}{c}
m-k \\
r-k
\end{array}\right) Q^{(r-k)}(z) R^{(k)}(z)
$$

is a joint covariant of $Q$ and $R$ called the $r$ th transvectant [17, Chapter 5].

We also form the following, fundamental, zero-weight rational covariants:

$$
J^{2}=\frac{T^{2}}{H^{3}}, \quad K=\frac{U}{H^{2}}
$$

Let $Q, \hat{Q} \in \mathcal{P}_{n}$ be two polynomials and let $H=H[Q], J=J[Q], K=K[Q]$ and $\hat{H}=H[\hat{Q}]$, $\hat{J}=J[\hat{Q}], \hat{K}=K[\hat{Q}]$ be the indicated covariants.

\footnotetext{
${ }^{5}$ For some values of the roots of $\mathrm{W}(\mathrm{z})$ the number of distinct elements may decrease. In fact it is an interesting question to understand when the roots are non-generic. We thank the referee for this observation.

${ }^{6}$ In the present setting we are interested in the case where $N=k \ell$ is the degree of the Wronskian covariant.
} 
Theorem 5.3 (Theorems 2.5 and 5.8 of [3]). The polynomials $Q, \hat{Q}$ are $\mathrm{PSL}_{2} \mathbb{C}$-equivalent if and only if both $H, \hat{H}=0$ or both $H, \hat{H} \neq 0$ and the following rational relations are compatible

$$
J^{2}(z)=\hat{J}^{2}(\hat{z}) \quad \text { and } \quad K(z)=\hat{K}(\hat{z}) .
$$

Furthermore, if (5.5) are consistent, then the resulting relation between $z$ and $\hat{z}$ is a fractional linear transformation.

Applying the criterion to $Q=W(U)$ and $\hat{Q}=W(\hat{U})$ with $N=k \ell=k(n+1-k)$ provides necessary condition for the equivalence of two polynomial subspaces $U, \hat{U} \in \mathcal{G}_{k} \mathcal{P}_{n}$. Indeed if $\operatorname{Stab} Q$ is trivial, the criterion is also sufficient. If $\operatorname{Stab} Q$ is a finite group, then the equivalence problem is reduced to the following finite procedure.

Theorem 5.4. Let $U, \hat{U} \in \mathcal{G}_{k} \mathcal{P}_{n}$ be polynomial subspaces. Suppose that $W(\hat{U})=g_{1} \cdot W(U)$ for some $g_{1} \in \mathrm{PSL}_{2} \mathbb{C}$. Then $U \sim \hat{U}$ if and only if there exists a projective transformation $g_{2} \in \operatorname{Stab} W(U)$ such that $\hat{U}=g_{1} \cdot g_{2} \cdot U$.

We refer to [3] for the classification of binary forms (polynomials) with finite, but non-trivial symmetry groups.

\subsection{The case of infinite symmetries}

In light of the above remarks, a full solution of the equivalence problem requires an understanding the class of subspaces $U \in \mathcal{G}_{k} \mathcal{P}_{n}$ with an infinite $\operatorname{Stab} U$. In this regard, let us recall the following helpful results from [3]. Throughout, $U \in \mathcal{G}_{k} \mathcal{P}_{n}$ is a polynomial subspace, $Q=W(U)$ is its Wronskian covariant, defined up to scalar multiple, and $N=k \ell=k(n+1-k)$.

Proposition 5.5. The following are equivalent:

- $Q(z)$ is a constant or $Q(z)=(a z+b)^{N}$ is a monomial of maximum degree.

- $H[Q]=0$;

- $\operatorname{Stab}(Q)$ is a 2-dimensional subgroup consisting of fractional linear transformations that fix exactly one point in $\hat{\mathbb{C}}$.

Proposition 5.6. The following are equivalent:

- $Q(z)=(a z+b)^{m}$ is a monomial where $m \neq 0, N$;

- $J^{2}[Q]$ is a constant;

- $\operatorname{Stab}(Q)$ is a 1-dimensional subgroup consisting of fractional linear transformations that fix exactly two points in $\hat{\mathbb{C}}$.

We use the above results to establish the following. Let us say that $U \in \mathcal{G}_{k} \mathcal{P}_{n}$ is a monomial subspace if $U=\operatorname{span}\left\{z^{\nu_{1}}, \ldots, z^{\nu_{k}}\right\}$, where $0 \leq \nu_{1}<\cdots<\nu_{k} \leq n$.

Theorem 5.7. The following are equivalent:

- $U$ is projectively equivalent to a monomial subspace;

- $Q=\mathrm{W}(U)$ has either one or two distinct roots;

- $H[Q]=0$ or $J^{2}[Q]$ is constant;

- $\operatorname{Stab}(Q)$ is infinite (of the type indicated in the above two propositions). 
Proof. We focus our attention on the most interesting of the above implications. First, suppose that $U$ is projectively equivalent to a monomial subspace. Since all of the above properties are $\mathrm{SL}_{2}$ equivariant, no generality is lost if we assume that $U$ is a monomial subspace with pivot sequence $\left\{\nu_{i}\right\}$. By Proposition 4.1, the Wronskian

$$
\mathrm{W}(U)(z)=\left[z^{m}\right], \quad \text { where } \quad m=\sum_{i} \nu_{i}-k(k-1) / 2
$$

is also a monomial. Hence, 0 and $\infty$ are the only roots. The other conclusions follow by Propositions 5.5 and 5.6.

Next, suppose that $\mathrm{W}(U)$ has one or two distinct roots. We will show that $U$ is a monomial subspace. By virtue of $\mathrm{SL}_{2}$-equivariance, no generality is lost in supposing that these roots are 0 and $\infty$ or just $\infty$ if there is only 1 root. Let $\left\{\nu_{i}\right\}$ and $\left\{\hat{\nu}_{i}\right\}$ be the corresponding 0 and $\infty$-pivot sequences. By Proposition 3.14, $\nu_{i} \leq \hat{\nu}_{i}$. Let

$$
\lambda_{i}=n-\nu_{i}-(k-i), \quad \hat{\lambda}_{i}=\hat{\nu}_{k+1-i}-(k-i)
$$

be the corresponding shape partitions. By Theorem 4.5,

$$
\operatorname{ord}_{0} Q=k \ell-\sum_{i} \lambda_{i}, \quad \operatorname{deg} Q=\sum_{i} \hat{\lambda}_{i}, \quad \operatorname{deg} Q-\operatorname{ord}_{0} Q=\sum_{i}\left(\hat{\nu}_{i}-\nu_{i}\right)
$$

Therefore, $\operatorname{deg} Q=\operatorname{ord}_{0} Q$ if and only if $\nu_{i}=\hat{\nu}_{i}$ for every $i$. By Corollary 3.15, the latter is true if and only if $U$ is spanned by monomials.

We emphasize that the above criterion is fully algorithmic. Given a basis of a polynomial subspace $U \in \mathcal{G}_{k} \mathcal{P}_{n}$, the algorithm requires us to calculate the Wronskian, and then calculate a finite number of differential invariants shown in (5.1)-(5.4). If $H=0$, then either $U=\mathcal{P}_{k-1}$ or there exists a $b \in \mathbb{C}$ such that

$$
U=\operatorname{span}\left\{(z-b)^{\ell}, \ldots,(z-b)^{n}\right\} .
$$

If $H \neq 0$, but $T^{2}$ is a constant multiple of $H^{3}$, then $U$ is projectively equivalent to a monomial subspace.

\subsection{Primitive subspaces}

We now consider two basic questions related to subspace equivalence. For $m<n$ we have the natural embedding $\mathcal{P}_{m} \subset \mathcal{P}_{n}$. It is natural to ask whether $\ell=n+1-k$ is the "true codimension", or whether it is possible to reduce the degree $n$ and the codimension $\ell$ by means of a fractional linear transformation. Dualizing, we obtain the following question: when does a subspace $U \subset \mathcal{P}_{n}$ contain a non-zero $n$th power $(a z+b)^{n} \in U$.

Definition 5.8. We say that $U$ is an imprimitive subspace if there exists a subspace $\hat{U} \sim U$ such that $\hat{U} \subset \mathcal{P}_{m}$ for some $m<n$. A primitive subspace is one for which no such $\hat{U}$ exists. We say that $U$ is strongly primitive if both $U$ and its apolar dual $U^{*}$ are primitive subspaces.

We also have the following characterizations of the above concepts ${ }^{7}$.

Proposition 5.9. A subspace $U \in \mathcal{G}_{k} \mathcal{P}_{n}$ is imprimitive if and only if $\operatorname{deg} U<n$ or if $\operatorname{ord}_{b} U>0$ for some $b \in \mathbb{C}$.

\footnotetext{
${ }^{7}$ In the interpretation of a polynomial subspace $U \in \mathcal{G}_{k} \mathcal{P}_{n}$ as a rational curve it is natural to regard the dual subspace $U^{*} \in \mathcal{G}_{\ell} \mathcal{P}^{n}$ as a linear series that "carves out" the given $U$. In this interpretation the condition of primitivity is equivalent to the condition that the series be basepoint-free; see $[14,6]$ for more details. We thank the referee for this observation.
} 
Definition 5.10. Let $U \in \mathcal{G}_{k} \mathcal{P}_{n}$ be a polynomial subspace. We say that $U$ contains a top power if $(z-b)^{n} \in U$ for some $b \in \mathbb{C}$ or if $1 \in U$.

Proposition 5.11. A polynomial subspace $U \in \mathcal{G}_{k} \mathcal{P}_{n}$ contains a top power if and only if the apolar dual space $U^{*}$ is imprimitive.

Proposition 5.12. A subspace $U \in \mathcal{G}_{k} \mathcal{P}_{n}$ is strongly primitive if and only if $\mathrm{M}(U, b)$ is nondegenerate $R R E F$ for all $b \in \hat{\mathbb{C}}$. Here, non-degenerate means having form (3.3).

To conclude this subsection, we present the following necessary, but not sufficient, condition for primitivity.

Proposition 5.13. Let $U \in \mathcal{G}_{k} \mathcal{P}_{n}$ be a subspace. If all roots of $\mathrm{W}(U)$ have multiplicities $<k$ then $U$ is primitive. If all roots have multiplicities $<\min (k, \ell)$, where $\ell=n+1-k$ is the codimension, then $U$ is strongly primitive.

Proof. If $U$ is imprimitive, then there exists a $b \in \hat{\mathbb{C}}$ such that $\mathrm{M}(U, b)$ is a degenerate RREF matrix of type (3.4) or (3.6). The width of the corresponding shape is $<\ell$. Hence, by Theorem 4.5 we have

$$
\operatorname{ord}_{b} \mathrm{~W}(U) \geq k \ell-k(\ell-1)=k .
$$

If $U^{*}$ is imprimitive, then by Theorems $3.10,3.13$, for some $b$, the RREF matrix $\mathrm{M}(U, b)$ is degenerate of type (3.5) or (3.6). The height of the corresponding shape is $<k$, and hence

$$
\operatorname{ord}_{b} \mathrm{~W}(U) \geq \ell \text {. }
$$

Therefore, if either $U$ or $U^{*}$ are imprimitive, then for all $b \in \hat{\mathbb{C}}$ we have

$$
\operatorname{ord}_{b} \mathrm{~W}(U) \geq \min (k, \ell) \text {. }
$$

\subsection{An example}

For the purposes of illustration let us consider the classification and equivalence of 2-dimensional subspaces of $\mathcal{P}_{3}$; i.e., $k=2, \ell=2$. In this case $d(2,2)=2$; this value corresponds to the classic result that generically, 4 lines in 3-dimensional space are simultaneously touched by exactly 2 lines. Consider two linearly independent polynomials $p_{1}, p_{2} \in \mathcal{P}_{3}$. Generically, the Wronskian $W\left(p_{1}, p_{2}\right)$ will have four distinct roots, call them $b_{1}, b_{2}, b_{3}, b_{4}$. These complex numbers are distinguished by the fact that the polynomial subspace $U=\operatorname{span}\left\{p_{1}, p_{2}\right\}$ contains exactly four (up to scalar multiple) polynomials with a double root. These polynomials have the form

$$
q_{i}(z)=r_{i}(z)\left(z-b_{i}\right)^{2}, \quad r_{i} \in \mathcal{P}_{2} .
$$

Now the subspaces

$$
L_{i}=\left\{r(z)\left(z-b_{i}\right)^{2}: r(z) \in \mathcal{P}_{2}\right\}
$$

represent 4 lines in 3-dimensional projective space $\mathcal{G}_{1} \mathcal{P}_{3}$. The subspace $U$ also represents a line. The polynomials $q_{1}, \ldots, q_{4}$ correspond to the 4 points where the line $[U]$ touches the four lines $\left[L_{1}\right], \ldots,\left[L_{4}\right]$. Since $d(2,2)=2$, in the generic case, there exists exactly one other subspace $\hat{U} \in \mathcal{G}_{2} \mathcal{P}_{3}$ with the same Wronskian. The lines $[U],[\hat{U}]$ are the unique lines that simultaneously touch $\left[L_{1}\right], \ldots,\left[L_{4}\right]$.

Let us now apply the above covariant analysis to describe the 2-dimensional polynomial subspaces of $\mathcal{P}_{3}$ with an infinite symmetry group. A generic subspace $U \in \mathcal{G}_{2} \mathcal{P}_{3}$ can be described as the span of polynomials

$$
p_{1}(z)=z^{3}+a_{11} z+a_{10}, \quad p_{2}(z)=z^{2}+a_{21} z+a_{20} .
$$


The corresponding Wronskian, and covariants are given by

$$
\begin{aligned}
W= & z^{4}+2 a_{21} z^{3}+\left(3 a_{20}-a_{11}\right) z^{2}-2 a_{10} z+\left(a_{11} a_{20}-a_{10} a_{21}\right) \\
= & z^{4}+b_{3} z^{3}+b_{2} z^{2}+b_{1} z+b_{0}, \\
H= & 3\left(8 b_{2}-3 b_{3}^{2}\right) z^{4}+12\left(6 b_{1}-b_{2} b_{3}\right) z^{3}+6\left(24 b_{0}-2 b_{2}^{2}+3 b_{1} b_{3}\right) z^{2} \\
& +12\left(6 b_{0} b_{3}-b_{1} b_{2}\right) z+3\left(8 b_{0} b_{2}-3 b_{1}^{2}\right) .
\end{aligned}
$$

A straight-forward calculation shows that $H=0$ if and only if

$$
a_{20}=-a_{11} / 3=-a_{10} / a_{21}=a_{21}^{2} / 4,
$$

or equivalently, iff

$$
W=\left(z+a_{21} / 2\right)^{4},
$$

in full accordance with Proposition 5.5.

Let us now describe all spaces projectively equivalent to a span of monomials. The case where $U$ is equivalent to the span of $\{1, z\}$ corresponds to $H=0$ and is described above. Let us now consider the other cases: $(i) U \sim \operatorname{span}\left\{z, z^{2}\right\}$; (ii) $U \sim \operatorname{span}\left\{1, z^{3}\right\}$; (iii) $U \sim \operatorname{span}\left\{1, z^{2}\right\}$. The other possible monomial subspaces are all projectively equivalent to one of these possibilities. For cases $(i)$ and $(i i)$ we have $J^{2}=0$; for case $(i i i) J^{2}=-16 / 9$. One can also arrive at the special values $J^{2}=0$ and $J^{2}=-16 / 9$ by applying the Gröbner basis algorithm to the principal ideal generated by $J^{2} H^{3}-T^{2}$ with $J$ as an extra variable.

Hence, for cases $(i)$ and $(i i)$ we must consider the condition $T=0$, where

$$
\begin{aligned}
T / 36= & \left(8 b_{1}-4 b_{2} b_{3}+b_{3}^{3}\right) z^{6}+2\left(16 b_{0}-4 b_{2}^{2}+2 b_{1} b_{3}+b_{2} b_{3}^{2}\right) z^{5} \\
& -5\left(4 b_{1} b_{2}-8 b_{0} b_{3}-b_{1} b_{3}^{2}\right) z^{4}-20\left(b_{1}^{2}-b_{0} b_{3}^{2}\right) z^{3}-5\left(8 b_{0} b_{1}+b_{1}^{2} b_{3}-4 b_{0} b_{2} b_{3}\right) z^{2} \\
& -2\left(16 b_{0}^{2}+b_{1}^{2} b_{2}-4 b_{0} b_{2}^{2}+2 b_{0} b_{1} b_{3}\right) z-b_{1}^{3}+4 b_{0} b_{1} b_{2}-8 b_{0}^{2} b_{3} .
\end{aligned}
$$

Straightforward calculations show that the general solution consists of two possibilities:

$$
\begin{aligned}
& a_{11}=a_{20}-a_{21}^{2}, \quad a_{10}=-a_{20} a_{21}, \quad W=\left(z^{2}+a_{21} z+a_{20}\right)^{2}, \\
& a_{11}=3\left(3 a_{20}-a_{21}^{2}\right), \quad a_{10}=3 a_{20} a_{21}-a_{21}^{3}, \quad W=\left(z^{2}+a_{21} z-a_{11} / 3\right)^{2} .
\end{aligned}
$$

Letting $r_{1}, r_{2}$ denote the double roots of the Wronskian, the two solutions of $T=0$ correspond to the following subspaces:

$$
\begin{aligned}
& U=\operatorname{span}\left\{\left(z-r_{1}\right)\left(z-r_{2}\right)^{2},\left(z-r_{1}\right)^{2}\left(z-r_{2}\right)\right\}, \\
& U=\operatorname{span}\left\{\left(z-r_{1}\right)^{3},\left(z-r_{2}\right)^{3}\right\} .
\end{aligned}
$$

The first of these is projectively equivalent to $\operatorname{span}\left\{z, z^{2}\right\}$, while the second is equivalent to $\operatorname{span}\left\{1, z^{3}\right\}$.

The analysis of case ( $i$ ii $)$ requires us to consider the solutions of the equation

$$
(16 / 9) H^{3}+T^{2}=0 .
$$

Calculation with a Gröbner basis package yields the following necessary condition:

$$
a_{11}+3 a_{20}=0
$$

Further computation reveals that the general solution is given by

$$
\begin{aligned}
& a_{11}=-b^{3} / 4+(3 / 4) b^{2} a_{21}-a_{21}^{3}, \quad a_{20}=-b^{2} / 4+a_{21}^{2} / 4, \\
& U=\operatorname{span}\left\{\left(z+\left(a_{21}+3 b\right) / 2\right)^{2}\left(z+\left(a_{21}-b\right) / 2\right),\left(z+\left(a_{21}-b\right) / 2\right)^{3}\right\},
\end{aligned}
$$


where $b$ is an arbitrary, non-zero complex parameter. Evidently, the above subspace is projectively equivalent to $\left\{1, z^{2}\right\}$.

As our final example, let us consider a polynomial subspace with a finite symmetry group. More specifically, let us consider subspaces with Wronskian $W=z^{4}-1$. Such a subspace must have a basis of the form

$$
U=\operatorname{span}\left\{(z-1)^{2}(z+a),(z+1)^{2}(z+b)\right\} .
$$

As expected, there are exactly two solutions:

$$
\begin{array}{lrl}
a=e^{\pi i / 3}, & b=e^{-2 \pi i / 3}, & U_{1}=\operatorname{span}\left\{z^{3}-\sqrt{3} i z, z^{2}-i / \sqrt{3}\right\}, \\
a=e^{-\pi i / 3}, & b=e^{2 \pi i / 3}, & U_{2}=\operatorname{span}\left\{z^{3}+\sqrt{3} i z, z^{2}+i / \sqrt{3}\right\} .
\end{array}
$$

Let us consider the question of whether $U_{1}$ is projectively equivalent to $U_{2}$. By construction, the two subspaces have the same Wronskian. We must therefore consider the projective symmetries of $W=z^{4}-1$. Applying the necessary formulas, we arrive at

$$
J^{2}(z)=-\frac{4\left(1+z^{4}\right)^{2}}{9 z^{4}}, \quad K(z)=-\frac{2\left(1+10 z^{4}+z^{8}\right)}{9 z^{4}} .
$$

Both of the rational function $J^{2}(z)-J^{2}(Z)$ and $K(z)-K(Z)$ have the same numerator, namely

$$
(z-Z)(z-i Z)(z+i Z)(z+Z)(-1+z Z)(-i+z Z)(i+z Z)(1+z Z) .
$$

Each of these factors defines a fractional linear transformation. The union of all such forms the octahedral symmetry group of the polynomial $z^{4}-1$. By Theorem 5.4 the subspaces $U_{1}$ and $U_{2}$ are projectively equivalent if and only if they are related by one of these transformations. Indeed, exactly half of these transformations, the subgroup consisting of

$$
z=Z, \quad z=1 / Z, \quad z=-Z, \quad z=-1 / Z
$$

are symmetries of $U_{1}$ and $U_{2}$. The other coset, consisting of the four transformations

$$
z=i Z, \quad z=i / Z, \quad z=-i Z, \quad z=-i / Z
$$

relate $U_{1}, U_{2}$. The two subspaces in question are projectively equivalent.

Remark: it is an interesting question to characterize and if possible to classify those polynomial subspaces whose projective symmetry group is strictly smaller than the symmetry group of the corresponding Wronskian.

\subsection{The problem of top powers}

Let us say that a $p \in \mathcal{P}_{n}$ is a top power if it is of the form $p(z)=(a z+b)^{n}$ where $(a, b) \neq(0,0)$. The following result is well-known in classical invariant theory [15].

Theorem 5.14 (Sylvester). Generically, a complex polynomial (binary form) having odd degree $n=2 m+1$ can be given as a sum of $m$ distinct top powers.

Indeed, determining the smallest number of $n$th powers required for the expression a given $p \in \mathcal{P}_{n}$ is a central problem of classical invariant theory. In this last subsection, we modify this question to the context of polynomial subspaces. First, we observe the following.

Proposition 5.15. Let $U \in \mathcal{G}_{k} \mathcal{P}_{n}$, where $k \leq n$, be a polynomial subspace. Then, $U$ contains at most $k$ top powers. 
Proof. Suppose that $U$ contains $k+1$ top powers. By applying an $\mathrm{SL}_{2}$ transformation, if necessary, without loss of generality, none of these is constant. By Proposition 5.11, every element of $U^{*}$ is divisible by $q(z)=\prod_{i=1}^{k+1}\left(z-b_{i}\right)$ where the $b_{i}$ are distinct. However, the subspace of $\mathcal{P}_{n}$ of polynomials divisible by $q(z)$ is $(n-k)$-dimensional. However, $\operatorname{dim} U^{*}=\ell=n+1-k-$ a contradiction.

Also observe that if $U \in \mathcal{G}_{k} \mathcal{P}_{n}$ contains $k$ distinct top powers, then these act as a basis for $U$. Geometrically, a subspace that admits a basis of top powers can be characterized as a secant flat of the rational normal curve [2]. Here, we focus on the following.

Question 5.16. How many top powers (up to scalar multiple) does a given polynomial subspace contain? Formulate a procedure to decide whether a polynomial subspace admits a basis of top powers.

The following procedure is one possible answer to this question. For a polynomial subspace $U$, let $U^{(j)}, j \geq 0$ denote the subspace spanned by the $j$ th derivatives of the elements of $U$.

Theorem 5.17. Let $U$ be a $k$-dimensional polynomial subspace with $n=\operatorname{deg} U$ and $\ell=n+1-k$ the codimension. Let $Q=\mathrm{W}\left(U^{(\ell-1)}\right)$. If $1 \notin U$, then $U$ admits a basis of top powers if and only if the following conditions hold:

(i) $Q$ has $k$ simple roots and

(ii) every $q \in U^{*}$ is divisible by $Q$.

Proof. Suppose that $U$ contains $k$ distinct top powers. Since $1 \notin U$ these have the form $\left(z-b_{i}\right)^{n}, i=1, \ldots, k$ for some $b_{i} \in \mathbb{C}$. Observe that

$$
Q\left(b_{i}\right)=\left.\mathrm{W}\left(\left(z-b_{1}\right)^{n-\ell+1}, \ldots,\left(z-b_{k}\right)^{n-\ell+1}\right)\right|_{z=b_{i}}=0, \quad i=1, \ldots, k
$$

and that $\operatorname{deg} Q=k$. Condition (i) is established. Condition (ii) follows by Proposition 5.11. Conversely, if conditions $(i)$ and (ii) hold then by Proposition 5.11, $U$ contains $k$ distinct top powers.

Mutatis mutandi we can formulate a version of the above theorem for the case where $1 \in U$. One merely has to replace $k$ with $k-1$ in condition $(i)$. Criterion $(i)$ can, in principle, be tested by calculating the discriminant of $Q$. Criterion (ii) requires an explicit basis of $U^{*}$. Such a basis can be obtained by means of Theorem 3.13. Therefore, the above theorem can serve as an algorithm for deciding the question of a top power basis. We also note that one direction of Theorem 5.17 follows from the more general Lemma 3.14 of [2].

As an interesting special case, we have the following. Let $U \in \mathcal{G}_{k} \mathcal{P}_{k}$ be a codimension 1 subspace and $\mathrm{W}(U) \in \mathcal{G}_{1} \mathcal{P}_{k}$ the corresponding Wronskian covariant. Counting $\infty$, let $1 \leq m \leq k$ be the number of distinct roots of $\mathrm{W}(U)$.

Proposition 5.18. The codimension 1 subspace $U$ contains $m$ top powers. In particular, $U$ admits a basis of top powers if and only if $\mathrm{W}(U)$ has simple roots only and $k-1 \leq \operatorname{deg} \mathrm{W}(U) \leq k$.

Proof. By Theorem 4.7, $U^{*}$ is 1-dimensional, spanned by $\mathrm{W}(U)$. The desired conclusion now follows by Propositions 5.9 and 5.11. In the generic case where $\mathrm{W}(U) \in \mathcal{P}_{k}$ has $k$ distinct roots, $U$ admits a basis of the form $\left(z-b_{i}\right)^{k}$ where $b_{1}, \ldots, b_{k}$ are the distinct roots of $\mathrm{W}(U)$. If $\operatorname{deg} W(U)=k-1$; i.e. if $\infty$ is a root of $\mathrm{W}(U)$, then the basis of top powers consists of 1 and $\left(z-b_{i}\right)^{k}$ where $b_{1}, \ldots, b_{k-1}$ are the distinct roots of $\mathrm{W}(U)$.

Our final contribution is the following conjecture. 
Proposition 5.19. If $U \in \mathcal{G}_{k} \mathcal{P}_{n}$ admits a basis of top powers then $W(U)$ is an $\ell$ th power with

$$
W(U)=W\left(U^{(\ell-1)}\right)^{\ell}
$$

where the equality is relative to $\mathcal{G}_{1} \mathcal{P}_{k \ell}$; i.e. up to non-zero scalar multiple.

Conjecture 5.20. Suppose that (5.6) holds and that $W\left(U^{(\ell-1)}\right)$ has $k$ distinct finite, simple roots. Then, $U$ admits a basis of top powers.

Proposition 5.18 establishes the conjecture in the case where the codimension $\ell=1$. We can also prove the conjecture for the case of codimension $\ell=2$. Since all of the roots of $W\left(U^{(1)}\right)$ are assumed to be finite, $1 \notin U$ and hence $U^{(1)}$ is $k$-dimensional. Let $b$ be one of the $k$ distinct roots of $W\left(U^{(1)}\right)$. From the assumption that $W(U)=W\left(U^{(1)}\right)^{2}$, it follows that $b$ is a double root of $W(U)$. Let $\boldsymbol{\lambda}$ be the $b$-shape of $U$ and $\boldsymbol{\nu}=\boldsymbol{\lambda}^{+}$the corresponding pivot sequence. If $\nu_{k}=n$, we are done; for this means that $(z-b)^{n} \in U$. Suppose then that $\nu_{k}<n$. Since $n=k+1$, this means that either $\nu_{k}=n-2=k-1$ or that $\nu_{k}=n-1=k$. If the former where true then, $\nu_{j}=j-1, j=1, \ldots, k$ and hence $b$ is not a root of $W(U)-$ a contradiction. Let's consider the case where $\nu_{k}=k$. Hence, $U$ contains a polynomial of the form $(z-b)^{k}+a(z-b)^{k+1}$. Hence $U^{(1)}$ contains a polynomial of the form $(z-b)^{k-1}+a_{1}(z-b)^{k}$. Let $\tilde{\nu}_{i}$ be the $b$-pivot sequence of $U^{(1)}$. Since $b$ is assumed to be a simple root of $W\left(U^{(1)}\right)$, we must have $\tilde{\nu}_{j}=j-1$ for $j=1, \ldots, k-1$ and $\tilde{\nu}_{k}=k$. However, we just argued that $U^{(1)}$ has a $b$-pivot in column $k-1$; this is a contradiction. Our claim is established.

\section{Acknowledgements}

Discussions with D. Gómez-Ullate and N. Kamran are gratefully acknowledged. P.C. was supported by an NSERC Undergraduate Summer Research Award. R.M. is supported by an NSERC discovery grant.

\section{References}

[1] Abdesselam A., Chipalkatti J., On the Wronskian combinants of binary forms, J. Pure Appl. Algebra 210 (2007), 43-61, math.AG/0507488.

[2] Chipalkatti J., Geramita A., On parameter spaces for Artin level algebras, Michigan Math. J. 51 (2003), 187-207, math.AG/0204017.

[3] Berchenko I., Olver P.J., Symmetries of polynomials, in Symbolic Computation in Algebra, Analysis, and Geometry (Berkeley, CA, 1998), J. Symbolic Comput. 29 (2000), 485-514.

[4] Eremenko A., Gabrielov A., The Wronski map and Grassmannians of real codimension 2 subspaces, Comput. Methods Funct. Theory 1 (2001), 1-25.

[5] Eastwood M., Michor P., Some remarks on the Plücker relations, in The Proceedings of the 19th Winter School "Geometry and Physics" (Srni, 1999), Rend. Circ. Mat. Palermo (2) Suppl. (2000), no. 63, 85-88, math.AG/9905090.

[6] Eisenbud D., Harris J., Divisors on general curves and cuspidal rational curves, Invent. Math. 74 (1983), 371-418.

[7] Finkel F., Kamran N., The Lie algebraic structure of differential operators admitting invariant spaces of polynomials, Adv. in Appl. Math. 20 (1998), 300-322, q-alg/9612027.

[8] Fulton W., Young tableaux. With applications to representation theory and geometry, London Mathematical Society Student Texts, Vol. 35, Cambridge University Press, Cambridge, 1997.

[9] Gómez-Ullate D., Kamran N., Milson R., Quasi-exact solvability and the direct approach to invariant subspaces, J. Phys. A: Math. Gen. 38 (2005), 2005-2019, nlin.SI/0401030.

[10] Gómez-Ullate D., Kamran N., Milson R., An extension of Böchner's problem: exceptional invariant subspaces, J. Approx. Theory, to appear, arXiv:0805.3376. 
[11] Gómez-Ullate D., Kamran N., Milson R., An extended class of orthogonal polynomials defined by a SturmLiouville problem, J. Math. Anal. Appl. 359 (2009), 352-367, arXiv:0807.3939.

[12] Griffiths P.A., Harris J., Principles of algebraic geometry, 2nd ed., John Wiley \& Sons, New York, 1994.

[13] Hilbert D., Theory of algebraic invariants, Cambridge University Press, Cambridge, 1993.

[14] Kharlamov V., Sottile F., Maximally inflected real rational curves, Mosc. Math. J. 3 (2003), 947-987, math.AG/0206268.

[15] Kung J.P.S., Rota G.-R., The invariant theory of binary forms, Bull. Amer. Math. Soc. (N.S.) 10 (1984), 27-85.

[16] Mukhin E., Tarasov V., Varchenko A., The B. and M. Shapiro conjecture in real algebraic geometry and the Bethe ansatz, Ann. Math. (2) 170 (2009), 863-881, math.AG/0512299.

[17] Olver P.J., Classical invariant theory, London Mathematical Society Student Texts, Vol. 44, Cambridge University Press, Cambridge, 1999.

[18] Olver P.J., Joint invariant signatures, Found. Comput. Math. 1 (2001), 3-67.

[19] Scherbak I., Rational functions with prescribed critical points, Geom. Funct. Anal. 12 (2002), 1365-1380, math.QA/0205168.

Scherbak I., A theorem of Heine-Stieltjes, the Wronski map, and Bethe vectors in the $s l_{p}$ Gaudin model, math.AG/0211377.

[20] Shafarevich I.R., Basic algebraic geometry, Springer-Verlag, Berlin - New York, 1977. 